\title{
Phase Transitions in a Single Supported Phospholipid Bilayer: Real-Time Determination by Neutron Reflectometry
}

\author{
Yuri Gerelli* \\ Institut Laue-Langevin, Grenoble, 38000, France
}

(Received 20 January 2019; published 20 June 2019)

\begin{abstract}
Time- and temperature-resolved neutron reflectometry allowed us to perform the real-time characterization of the structural changes taking place across phase transitions in solid supported-lipid bilayers (SLBs). We identified the presence of an isothermal phase transition, characterized by a symmetrical rearrangement of lipid molecules in both bilayer leaflets, followed by the main thermotropic phase transition, and characterized by an independent melting of the two leaflets. We demonstrated that the presence of a substrate increases the enthalpy of melting by the same amount for both SLB leaflets with respect to the values reported for freestanding bilayers. These results are highly relevant for the further understanding of cooperative structural dynamics in SLBs and for the investigation of thermally activated processes such as the transmembrane lipid translocation (flip flop).
\end{abstract}

DOI: 10.1103/PhysRevLett.122.248101

Lipid bilayers are thin films made of two layers of lipid molecules. Solid-supported lipid bilayers (SLBs) are widely used tools in biological and technological studies for the investigation of interactions and molecular processes involved in cell function [1,2], disease [3], and for sensing applications $[4,5]$. Coexistence of ordered and disordered domains, structural and dynamic coupling between leaflets, and the structural responses to changes in the environmental parameters, such as temperature, are of fundamental interest in all of the above mentioned fields. Moreover, SLBs are one of the prototypes of natural selfassembling systems.

Phase transitions in lipid bilayers have been widely studied in solution aggregates, such as vesicles [6-9], or in solid-supported stacks of hundreds of bilayers in controlled humidity environments [10]. However, a lack of information still exists for systems with low-dimensionality such as SLBs, where the effects of supporting interfaces, finite size, and planarity might play important roles.

Phase transitions in single SLBs have been addressed mainly by means of AFM (atomic force microscopy) [11-14], SFG-VS (sum-frequency generation vibrational spectroscopy) [14], MD (molecular dynamics) simulations [15], and calorimetry [16]. The main common result is a substantial broadening of the gel-to-fluid phase transition (PT) with respect to that observed in freestanding bilayers in solution. In some cases, AFM and calorimetry

Published by the American Physical Society under the terms of the Creative Commons Attribution 4.0 International license. Further distribution of this work must maintain attribution to the author(s) and the published article's title, journal citation, and DOI. measurements have shown a decoupling between the PT behavior of the portion of the SLB facing the solid substrate (proximal leaflet) and the one closer to the water subphase (distal leaflet) $[13,14,16]$. In particular, Xie and co-workers [11] reported that the gel-to-fluid PT in a single DMPC (1,2-dimyristoyl-sn-glycero-3-phosphocholine) SLB is characterized by the formation and subsequent growth of fluid domains as the temperature is increased; coexistence between gel and fluid phases was observed in the central part of the PT, until the growing domains entirely replaced the starting phase. Based on this observation on the SLB surface morphology, they concluded that this behavior was linked to structural changes in the vertical direction normal to the bilayer surface. Wu and co-workers [14] were able to further characterize the structural changes taking place between 20 and $60^{\circ} \mathrm{C}$ in a DPPC (1,2-dipalmitoyl-snglycero-3-phosphocholine) SLB by means of AFM. They confirmed that the melting process is mediated by the growth of nanosized domains. Moreover, by evaluating the changes in bilayer thickness, they hypothesized the subsequent and individual melting of the proximal and distal leaflets. They concluded that the PT of the distal leaflet occurred in the 20 to $40^{\circ} \mathrm{C}$ temperature interval while that of the proximal leaflet started at $40^{\circ} \mathrm{C}$ to be completed at $60^{\circ} \mathrm{C}$. However, information on the internal structure of the bilayers was not directly accessible. The drawback of AFM techniques is in fact the limited sensitivity to the internal structure of a sample. Moreover, AFM measurements are characterized by a long measuring time and by a very high spatial resolution. However, it is restricted to small portions of the sample, typically on the order of $100 \mu \mathrm{m}^{2}$.

This Letter describes how time- and temperatureresolved (TTR) neutron reflectometry (NR) can provide 
a real-time direct characterization of the internal structural changes taking place in SLBs across their phase transitions. We demonstrate how this method can be used to obtain direct information on the melting behavior of the proximal and distal leaflets in SLBs. Time-resolved neutron scattering techniques have been proven to be optimal tools for the real-time characterization of structural processes in lipid assemblies [17]. Their combination to temperatureresolved measurements has the great advantage of providing thermodynamic and kinetic information on broad time and temperature intervals for a single sample. Indeed, sample reproducibility represents a big drawback in soft matter and biophysics and it is a limiting factor for the investigation of SLBs [18]. In the present case, the use of a single sample allowed us to probe more thermodynamic conditions than those typically probed with the approaches used so far [19].

For this Letter, we used DPPC and its partially deuterated homolog $d_{75}$ DPPC (1,2-dipalmitoyl-d62-sn-glycero3-phosphocholine-1,1,2,2-d4- $N, N, N$-trimethyl-d9) as representative phospholipid molecules. These phospholipids are widely used in physical and biophysical studies [20,21] as well as in the previously reported AFM and SFG-VS experiments $[12,14]$. In solution, DPPC vesicles exhibit distinct, well-defined phases such as the crystal ( $L_{c}$, for $T<14{ }^{\circ} \mathrm{C}$ ), gel $\left(L_{\beta}\right.$, for $\left.14<T<35^{\circ} \mathrm{C}\right)$, ripple $\left(P_{\beta}\right.$, for $\left.35<T<41{ }^{\circ} \mathrm{C}\right)$, and fluid $\left(L_{\alpha}\right.$, for $\left.T>41^{\circ} \mathrm{C}\right)$ phases [22]. In the gel phase, phospholipid molecules are highly ordered and packed in a regular lattice; the hydrophobic acyl chains of the molecules are stretched, increasing the bilayer thickness. In the fluid phase, bilayers are characterized by an irregular packing, lower thickness and larger average molecular area $[23,24]$. The intermediate ripple phase, generally observed in multibilayer structures, is characterized by the appearance of a periodic undulated bilayer structure [25]. The enthalpy of the gel-to-ripple transition is very small compared to that of the ripple-tofluid transition and the appearance of the ripple phase depends on the thermal history of the sample. For these reasons, the main transition is usually given as the gel-tofluid transition. The NR measurements reported in the present Letter were performed on the D17 neutron reflectometer [26] (Institut Laue-Langevin, Grenoble) using a divergent beam configuration [27] in a temperature range from 25 to $60^{\circ} \mathrm{C}$, i.e., for the temperature intervals in which $L_{\beta} \rightarrow P_{\beta}$ and $P_{\beta} \rightarrow L_{\alpha}$ phase transitions were expected. Two angular configurations $\left(\theta=0.8^{\circ}\right.$ and $\left.\theta=3.0^{\circ}\right)$ were used for the characterization of the sample before $\left(25^{\circ} \mathrm{C}\right)$ and after $\left(60^{\circ} \mathrm{C}\right)$ the main phase transition, while an intermediate configuration $\left(\theta=2.0^{\circ}\right)$ was used during TTR-NR measurements. For all these configurations, the neutron beam illuminated the interface through the solid substrate with a constant footprint, covering the $50 \%$ of the sample surface. For details about the experimental conditions see the Supplemental Material [28]. DPPC and $d_{75}$ DPPC SLBs were deposited on polished silicon crystals $\left(8 \times 5 \mathrm{~cm}^{2}\right.$ surface, $1.5 \mathrm{~cm}$ thick) by Langmuir-Blodgett and Langmuir-Schaefer deposition techniques [29] from monolayers prepared at the air-water interface at a high surface pressure $(\pi=50 \mathrm{mN} / \mathrm{m})$. The initial and final structure of the bilayers was characterized using the contrast variation method [30] at 25 and $60^{\circ} \mathrm{C}$ in the gel and fluid phases, respectively. In order to apply the contrast variation method, the $\mathrm{H}_{2} \mathrm{O}: \mathrm{D}_{2} \mathrm{O}$ ratio in the subphase was changed systematically between $\mathrm{D}_{2} \mathrm{O}, \mathrm{H}_{2} \mathrm{O}$ and a $38: 62$ $\mathrm{H}_{2} \mathrm{O}: \mathrm{D}_{2} \mathrm{O}$ mixture. Datasets collected for DPPC and $d_{75}$ DPPC bilayers at the same temperature were modeled using a common set of structural parameters (global fit) while applying molecular constraints for the conservation of the molecular area as described in literature [31]. This allowed us to accurately determine two sets of parameters describing the initial and final states of the bilayer, which resulted to be in agreement with already published data [32,33]. A sketch of a SLB is given in Fig. 1 (for more details about the modeling, see the Supplemental Material [28]). An important parameter obtained from these analysis is the amount of water in the hydrophobic region of the bilayer. This parameter is a measure the amount of defects in the SLB films; the result was for it to be reduced to a negligible amount, i.e., lower than $1 \%$ by volume (see Table S1 and Fig. S2 in the Supplemental Material [28]). This is a crucial feature since defects have been shown to impact the structural dynamics in SLBs: for example, they make transbilayer migration of lipid material faster [34,35] and they can also affect the phase transition behavior.

TTR-NR data were collected for DPPC and $d_{75}$ DPPC samples in $\mathrm{D}_{2} \mathrm{O}$. In this case, the analysis has been

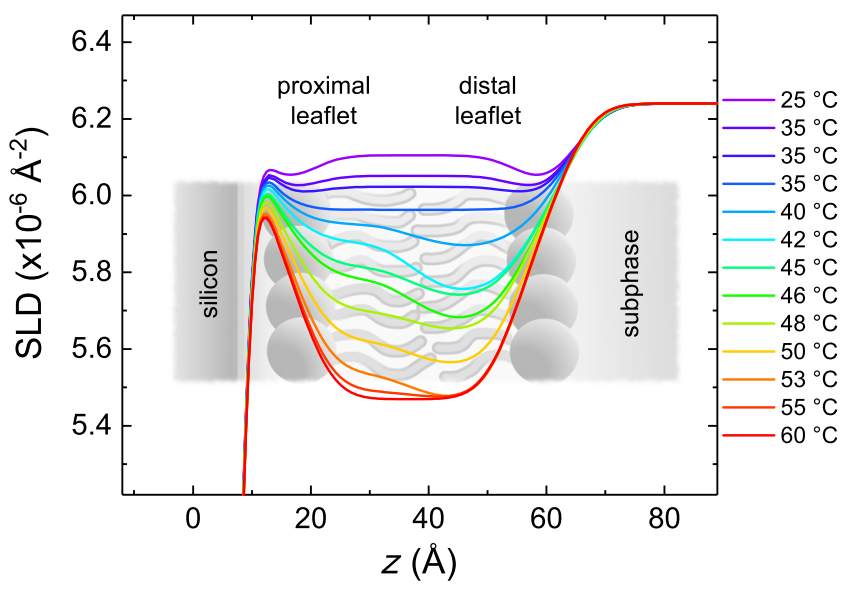

FIG. 1. Selected SLD profiles (view enlargement on the bilayer region) describing the structure of the $d_{75}$ DPPC bilayer at different stages of evolution. The temperatures corresponding to the curves are indicated in the legend (temperature increases top to bottom). The decrease in the SLD of the hydrophobic region (centered at $z \approx 40 \AA$ ) at constant temperature is visible by comparing the profiles obtained at $35^{\circ} \mathrm{C}$. For higher temperatures, the early melting of the distal leaflet is clearly visible. 
performed on the individual datasets. The NR signal is dominated by the difference in scattering length density [30] (SLD or $\rho$ ) between regions of the sample and the surrounding bulk phases, $\mathrm{D}_{2} \mathrm{O}$ and silicon in the present case. The SLD of hydrogenated phospholipids tails is close to zero, and therefore its relative variation upon PT is minimal. For this reason, the highest sensitivity to temperature-induced changes was achieved by using $d_{75}$ DPPC. During TTR-NR measurements, the temperature was changed stepwise, with $5^{\circ} \mathrm{C}$ steps every 22 minutes from 25 to $40^{\circ} \mathrm{C}$ and with $1{ }^{\circ} \mathrm{C}$ steps every 15 minutes from 40 to $60{ }^{\circ} \mathrm{C}$. Thermal equilibrium (within $\pm 0.1{ }^{\circ} \mathrm{C}$ ) was reached in less than 2 minutes for every step and reflectivity data corresponding to nonequilibrated states were not included in the analysis. The temperature-time curve used is shown in the Supplemental Material [28]. It is important to remark that the difference in the scanning rate between the two intervals was a consequence of the fact that the experiment was designed to focus on the main PT. During TTR-NR measurements, reflectivity data were recorded with 30 second frames. This duration was judged sufficient to provide data with sufficient statistics for the structural analysis (see Supplemental Material for examples of TTRNR data [28]). Each frame was analyzed using two approaches: once assuming a symmetric bilayer structure and once assuming an asymmetric structure with different properties for the two leaflets. As suggested by AFM measurements, during the main PT $\left(P_{\beta} \rightarrow L_{\alpha}\right.$ in the present Letter) the coexistence of domains of the two phases is expected, either across the bilayer [11] or for every leaflet independently [14]. As demonstrated by AFM domains in DPPC, SLBs are expected to be nanosized, and are therefore much smaller than the $\approx 10 \mu \mathrm{m}^{2}$ coherence area of the neutron beam [36] used for the experiments reported in this letter. NR experiments were not conclusive on the presence of nanosized gel or fluid domains and the possibility that the bilayer would undergo a continuous and uniform transformation [37] was taken into account. In both cases, the mathematical descriptions of the SLB structure, as seen by NR, are equivalent. In fact, in the presence of domains much smaller than the coherence length, the total specular reflectivity is equal to the reflectivity of a uniform sample averaged over the illuminated area. Such a reflectivity was computed from a set of average parameters defined according to the generalized formula

$$
X_{j}=\left(1-\Phi_{i, f}\right) X_{j}^{i}+\Phi_{i, f} X_{j}^{f},
$$

where $X_{j}$ is a stand-in parameter for the tail thicknesses $\left(t_{t 1}\right.$ and $\left.t_{t 2}\right)$, the water volume fraction in the headgroups $\left(f_{w}^{h 1}\right.$, $\left.f_{w}^{h 2}\right)$ and tails $\left(f_{w}^{t 1}, f_{w}^{t 2}\right)$ and the SLD of the headgroups $\left(\rho_{h 1}, \rho_{h 2}\right)$ and tails $\left(\rho_{t 1}, \rho_{t 2}\right)$. The suffixes $i$ and $f$ in Eq. (1) indicate the values of a given parameter before and after the transition. In the case of the gel-to-fluid PT the corresponding values of reference are given in Table S1 in the
Supplemental Material [28]. $\Phi_{i, f}$ in Eq. (1) can be interpreted as the amount of domains of the final phase present in the SLB at the nanoscale or, equivalently, as a parameter describing the extent of a continuous transition. Among fit parameters, the SLD of the tail regions $\rho_{t 1}$ and $\rho_{t 2}$ turned out to be the most sensitive to the changes induced by the phase transitions. For this reason, values of $\Phi_{i, f}$ were actually obtained as

$$
\Phi_{i, f}=\frac{\rho_{t}-\rho_{t}^{i}}{\rho_{t}^{f}-\rho_{t}^{i}} .
$$

Through Eqs. (1) and (2), an average reflectivity, to be fitted to experimental data, was calculated. This led to the optimization of the $\Phi_{i, f}$ parameters. For examples of TTR fits, see the Supplemental Material [28]. Selected SLD profiles obtained by the analysis of TTR-NR data are shown in Fig. 1. The full set of SLD profiles are shown as a color map in the Supplemental Material [28]. It is worth recalling that SLD profiles are directly related to the distribution of the different molecular species within the SLB, and they therefore represent a structural description of the bilayer. In the initial $\left(t \leq 5000 \mathrm{~s}, T \leq 40^{\circ} \mathrm{C}\right)$ and final ( $t>15000 \mathrm{~s}, T \geq 55^{\circ} \mathrm{C}$ ) regimes, i.e., at the beginning and end of the kinetics, the SLD profiles turned out to be completely symmetric. While in the final regime, once the bilayer melting was completed, the structure of the bilayer was symmetric and did not evolve further, between 30 and $40^{\circ} \mathrm{C}$ it changed continuously as a function of both time and temperature, while maintaining its symmetry. This continuous changes are visible in Fig. 1, where three SLD profiles measured at different times but at a constant temperature $\left(35^{\circ} \mathrm{C}\right)$ are compared. Such a behavior is compatible with an isothermal PT that maintains the symmetry of the bilayer. This clear time-evolution at a constant temperature is also reported in Fig. 2(a). Here, the transition extent parameter was named $\Phi_{\beta, \beta}$ because the observed transformation took place in a temperature interval corresponding to that of the $L_{\beta} \rightarrow P_{\beta}$ transition observed for DPPC bilayers [25,38]. The current data cannot be conclusive on the origin of such a process never previously observed in a single SLB. However, it is worth noting that this transition takes place in the same exact temperature interval reported by $\mathrm{Wu}$ and co-workers for the individual melting of the distal leaflets [14]. The melting model described in Ref. [14] was tested against our data and its likelihood turned out to be extremely low, suggesting that we could discard this scenario with high confidence. We could therefore identify an unexpected isothermal transition that can be probably related to the gelto-ripple PT that was never observed in single SLBs. Further experiments are necessary to better characterize this process and to clarify its origin. For temperatures close to that of the main PT in solution (assumed here to be $P_{\beta} \rightarrow L_{\alpha}$ ), the time-dependence observed in the pretransition disappears: $\Phi_{\beta, \alpha}$ remained constant for both leaflets at 

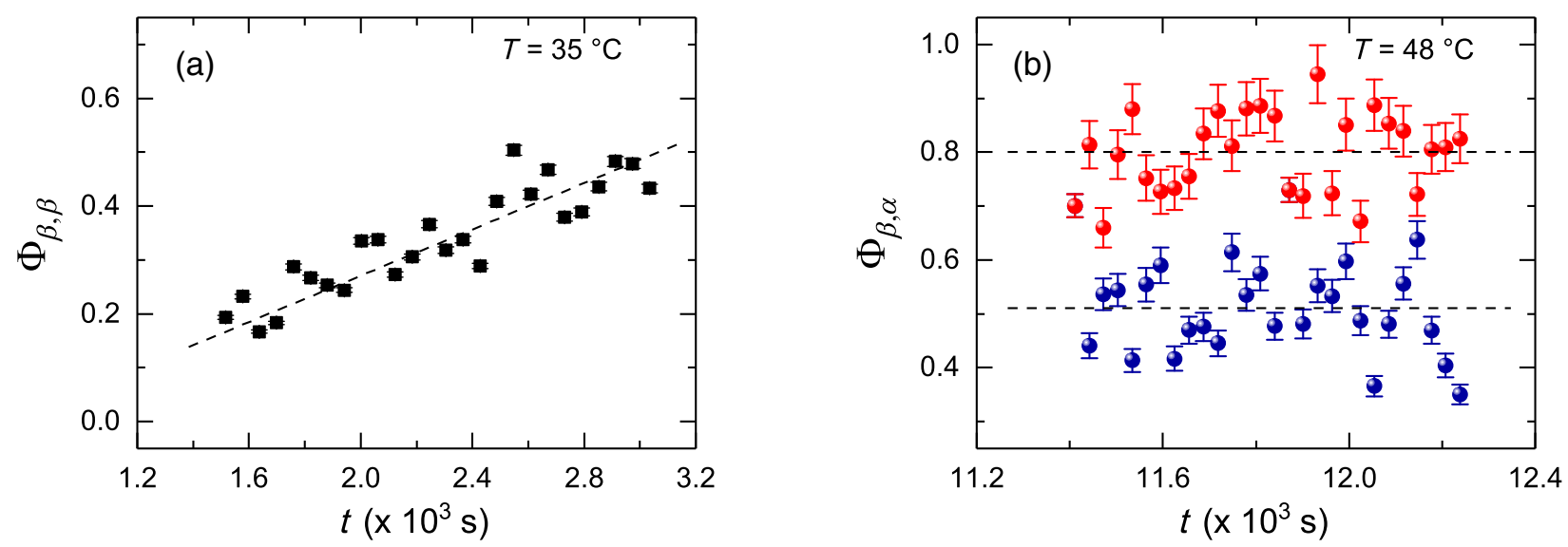

FIG. 2. Time-evolution of the parameters describing the extent of the $L_{\beta} \rightarrow P_{\beta}\left[\Phi_{\beta, \beta}, T=35^{\circ} \mathrm{C}\right.$, panel (a) $]$ and of the $P_{\beta} \rightarrow L_{\alpha}\left[\Phi_{\beta, \alpha}\right.$, $T=48^{\circ} \mathrm{C}$, panel (b)] transitions. $\Phi_{\beta, \beta}$ was calculated from a symmetric model while $\Phi_{\beta, \alpha}$ values were obtained assuming decoupled leaflets [proximal leaflet, black symbols (blue); distal leaflet: gray symbols (red)].

a given temperature, as expected for a thermotropic transition [Fig. 2(b)]. However, $\Phi_{\beta, \alpha}$ exhibited a marked temperature dependence, which was different for the proximal and distal leaflets: the structure of the bilayer was clearly asymmetric between 41 and $52^{\circ} \mathrm{C}$. In this regime, the proximal leaflet experienced a delayed melting, characterized by $\mathrm{a}+4{ }^{\circ} \mathrm{C}$ shift with respect to the melting curve of the distal leaflet. This feature is visible in the inset in Fig. 3 where the temperature evolution of $\Phi_{\beta, \alpha}$ for the two leaflets is compared. Each point represents the average value resulting from the analysis of $25 \mathrm{NR}$ curves measured as a function of time while keeping the temperature constant. The main thermotropic transition was analyzed in detail using a classical van 't Hoff approach, also used for the interpretation of AFM and SFG-VS data $[11,13,14]$. Two equilibrium constants, $k_{\text {eq1 }}$ and $k_{\text {eq2 }}$, for the proximal and distal leaflets were defined as

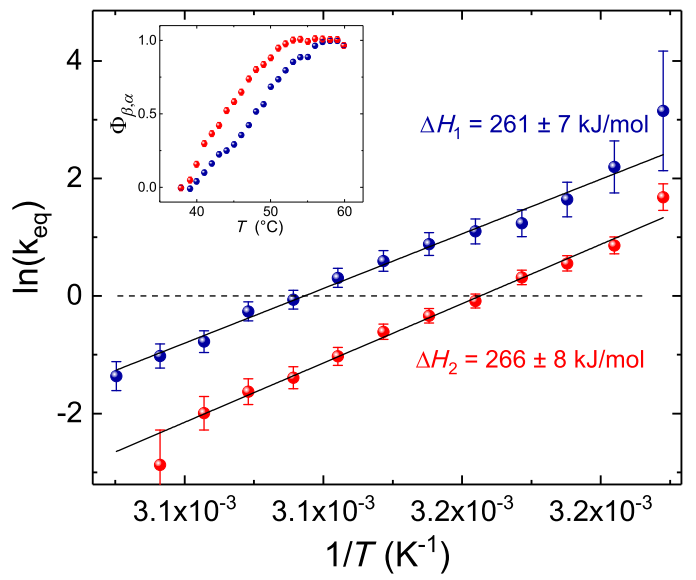

FIG. 3. Van 't Hoff plot describing the extent of the $P_{\beta} \rightarrow L_{\alpha}$ transition for proximal (black symbols) and distal (gray symbols) leaflets. The enthalpy values obtained from the linear fits are shown in the graph. The dashed line indicates the midpoint of the transition. Inset: temperature dependence plot for $\Phi_{\beta, \alpha}$ (proximal leaflet, black symbols; distal leaflet: gray symbols).

$$
k_{\mathrm{eq}}=\frac{1-\Phi_{\beta, \alpha}}{\Phi_{\beta, \alpha}}=e^{(-\Delta H / R T)+(\Delta S / R)}
$$

The transition enthalpy, calculated from the linear fit of $\ln \left(k_{\text {eq }}\right)$ vs $1 / T$ according to Eq. (3), resulted $\Delta H_{1}=261 \pm$ $7 \mathrm{~kJ} / \mathrm{mol}$ and $\Delta H_{2}=266 \pm 8 \mathrm{~kJ} / \mathrm{mol}$ for the proximal and distal leaflets respectively (the corresponding entropy values were $\Delta S_{1}=814 \pm 24 \mathrm{~J} \mathrm{~mol}^{-1} \mathrm{~K}^{-1}$ and $\Delta S_{2}=840 \pm 25 \mathrm{~J} \mathrm{~mol}^{-1} \mathrm{~K}^{-1}$ ). These enthalpy values are larger than those commonly reported for large uni- and multilamellar vesicles of DPPC $(\Delta H \approx 36 \mathrm{~kJ} / \mathrm{mol}$ [6], $\Delta H \approx 30 \mathrm{~kJ} / \mathrm{mol}$ [39]) and this can be interpreted as an effect of the interaction with the substrates. Similar values were found for the distal leaflet in DPPC SLBs by AFM measurements $[13,14]$. On the other hand, the enthalpy values found in the present Letter for the proximal leaflet are substantially lower than those resulting from the interpretation of AFM images $\left(\Delta H_{1} \approx 800-1000 \mathrm{~kJ} / \mathrm{mol}\right.$ in Refs. $[13,14])$. More importantly, we did not observe any substantial difference in the melting transition enthalpy of the two leaflets. The only trace of structural asymmetry induced by the substrate between the two leaflets is the $+4{ }^{\circ} \mathrm{C}$ shift between the melting curves of proximal and distal leaflets. In conclusion, the newly developed TTR-NR method allowed us to simultaneously investigate the timeand temperature-dependence of the structural changes taking place in SLBs undergoing a phase transition. Contrary to other approaches used for this type of characterization, TTR-NR measurements can probe directly the internal structure of SLBs and obtain therefore direct information about the changes taking place in both leaflets. Indeed, this method allowed us to distinguish two different phase transitions that, without looking at constant temperature data, would appear as a single one because of their broadening and consecutive overlap. The first isothermal transition was completely unexpected and further investigations will be necessary for a more detailed characterization. AFM and SFG-VS measurements showed already the presence of a structural rearrangement process in the 
same temperature interval but, because of the lack of information on the internal SLB structure, this process was interpreted as the independent melting of the distal leaflet. We have demonstrated that this transition differs from the melting one and that takes place simultaneously in both SLB leaflets. The main transition to the fluid phase was broader than the one observed in freestanding bilayers in agreement with AFM and SFG-VS results. During this transition, the bilayer structure was characterized by a minor, but not negligible, degree of asymmetry induced by the presence of a solid substrate. The energy required to melt the two leaflets was larger than that required to melt DPPC vesicles in solution, but it did not vary between proximal and distal leaflets, indicating a minor effect of the solid substrate. These results are essential to better understand the structural behavior in systems at low dimensionality, such as SLBs, and to tackle new, challenging problems related to their structural dynamics as, e.g., the lipid flip flop.

The author thanks the Institut Laue-Langevin for the awarded beamtime (DOIs: 10.5291/ILL-DATA.9-13-759 and 10.5291/ILL-DATA.9-13-742) and the Partnership for Soft Condensed Matter for the access to Langmuir troughs, ellipsometer and sample preparation facilities as well as for providing the deuterated phospholipids used in this Letter. For critical suggestions and discussion, I thank Lionel Porcar and Dominic Hayward.

*gerelli@ill.fr

[1] B. R. Olden, C. R. Perez, A. L. Wilson, I. I. Cardle, Y.-S. Lin, B. Kaehr, J. A. Gustafson, M. C. Jensen, and S. H. Pun, Adv. Healthcare Mater. 8, e1801188 (2019).

[2] B. Josey, F. Heinrich, and M. Lösche, Biophys. J. 114, 258a (2018).

[3] A. Martel, L. Antony, Y. Gerelli, L. Porcar, A. Fluitt, K. Q. Hoffmann, I. Kiesel, M. Vivaudou, G. Fragneto, and J. J. de Pablo, J. Am. Chem. Soc. 139, 137 (2017).

[4] E. T. Castellana and P. S. Cremer, Surf. Sci. Rep. 61, 429 (2006).

[5] M. P. Jonsson, P. Jönsson, A. B. Dahlin, and F. Höök, Nano Lett. 7, 3462 (2007).

[6] S. Mabrey and J. M. Sturtevant, Proc. Natl. Acad. Sci. U.S.A. 73, 3862 (1976).

[7] D. Small and D. Hanahan, Handbook of Lipid Research (Plenum Press, London, 1986), Vol. 4.

[8] M. C. Wiener, S. Tristram-Nagle, D. A. Wilkinson, L. E. Campbell, and J.F. Nagle, Biochim. Biophys. Acta Biomembr. 938, 135 (1988).

[9] R. L. Biltonen and D. Lichtenberg, Chem. Phys. Lipids 64, 129 (1993).

[10] J. F. Nagle and S. Tristram-Nagle, Biochim. Biophys. Acta 1469, 159 (2000).

[11] A. F. Xie, R. Yamada, A. A. Gewirth, and S. Granick, Phys. Rev. Lett. 89, 246103 2002).
[12] Z. V. Leonenko, E. Finot, H. Ma, T. E. S. Dahms, and D. T. Cramb, Biophys. J. 86, 3783 (2004).

[13] D. Keller, N. B. Larsen, I. M. Møller, and O. G. Mouritsen, Phys. Rev. Lett. 94, 025701 (2005).

[14] H.-L. Wu, Y. Tong, Q. Peng, N. Li, and S. Ye, Phys. Chem. Chem. Phys. 18, 1411 (2016).

[15] T. Schubert, E. Schneck, and M. Tanaka, J. Chem. Phys. 135, 055105 (2011).

[16] L. Yang and H. W. Huang, Science 297, 1877 (2002).

[17] M. Nakano, M. Fukuda, T. Kudo, H. Endo, and T. Handa, Phys. Rev. Lett. 98, 238101 (2007).

[18] J. Kurniawan, J. F. Ventrici De Souza, A. T. Dang, G. Y. Liu, and T. L. Kuhl, Langmuir 34, 15622 (2018).

[19] Y. Gerelli, L. Porcar, L. Lombardi, and G. Fragneto, Langmuir 29, 12762 (2013).

[20] N. Kučerka, J. F. Nagle, J. N. Sachs, S. E. Feller, J. Pencer, A. Jackson, and J. Katsaras, Biophys. J. 95, 2356 (2008).

[21] H. P. Wacklin, Langmuir 27, 7698 (2011).

[22] H. W. Meyer, K. Semmler, W. Rettig, W. Pohle, A. S. Ulrich, S. Grage, C. Selle, and P. J. Quinn, Chem. Phys. Lipids 105, 149 (2000).

[23] M. C. Wiener, R. M. Suter, and J. F. Nagle, Biophys. J. 55, 315 (1989).

[24] R. Koynova and B. Tenchov, in Wiley Encyclopedia of Chemical Biology, edited by T. P. Begley (John Wiley \& Sons, Inc., Hoboken, 2008), pp. 1-15.

[25] T. Kaasgaard, C. Leidy, J. H. Crowe, O. G. Mouritsen, and K. Jørgensen, Biophys. J. 85, 350 (2003).

[26] T. Saerbeck, R. Cubitt, A. Wildes, G. Manzin, K. H. Andersen, and P. Gutfreund, J. Appl. Crystallogr. 51 (2018).

[27] R. Cubitt, T. Saerbeck, R. A. Campbell, R. Barker, and P. Gutfreund, J. Appl. Crystallogr. 48, 2006 (2015).

[28] See Supplemental Material at http://link.aps.org/ supplemental/10.1103/PhysRevLett.122.248101 for more details on the modeling approach, on the instrumental configuration, and on the structural characterization of the initial and final bilayer structure.

[29] M. C. Petty and W. A. Barlow, Film Deposition, in Langmuir-Blodgett Film, edited by G. Roberts (Springer US, Boston, 1990), pp. 93-132.

[30] T. L. Crowley, E. M. Lee, E. A. Simister, and R. K. Thomas, Phys. B 173, 143 (1991).

[31] Y. Gerelli, J. Appl. Crystallogr. 49, 330 (2016).

[32] E. B. Watkins, C. E. Miller, D. J. Mulder, T. L. Kuhl, and J. Majewski, Phys. Rev. Lett. 102, 238101 (2009).

[33] M. Belička, Y. Gerelli, N. Kučerka, and G. Fragneto, Soft Matter 11, 6275 (2015).

[34] Y. Gerelli, L. Porcar, and G. Fragneto, Langmuir 28, 15922 (2012).

[35] D. Marquardt, F. A. Heberle, T. Miti, B. Eicher, E. London, J. Katsaras, and G. Pabst, Langmuir 33, 3731 (2017).

[36] B. Dorner and A. R. Wildes, Langmuir 19, 7823 (2003).

[37] S. Tristram-Nagle and J. F. Nagle, Chem. Phys. Lipids 127, 3 (2004).

[38] M. P. Hentschel and F. Rustichelli, Phys. Rev. Lett. 66, 903 (1991).

[39] H. Ebel, P. Grabitz, and T. Heimburg, J. Phys. Chem. B 105, 7353 (2001). 